\title{
KORN INEQUALITY AND DIVERGENCE OPERATOR: COUNTEREXAMPLES AND OPTIMALITY OF WEIGHTED ESTIMATES
}

\author{
GABRIEL ACOSTA, RICARDO G. DURÁN, AND FERNANDO LÓPEZ GARCÍA \\ (Communicated by Walter Craig)
}

\begin{abstract}
The Korn inequality and related results on solutions of the divergence in Sobolev spaces have been widely studied since the pioneering works by Korn and Friedrichs. In particular, it is known that this inequality is valid for Lipschitz domains as well as for the more general class of John domains. On the other hand, a few known counterexamples show that those results are not valid for certain bounded domains having external cusps.

The goal of this paper is to give very simple counterexamples for a class of cuspidal domains in $\mathbb{R}^{n}$. Moreover, we show that these counterexamples can be used to prove the optimality of recently obtained results involving weighted Sobolev spaces.
\end{abstract}

\section{INTRODUCTION}

This paper deals with two related results which are basic in the analysis of problems in continuum mechanics, namely, the existence of solutions of the divergence in Sobolev spaces and the so-called Korn inequality.

Let us recall these two results. For a vector field $\mathbf{v} \in W^{1, p}(\Omega)^{n}, \varepsilon(\mathbf{v})$ denotes the symmetric part of the differential matrix of $\mathbf{v}$, namely,

$$
\varepsilon_{i j}(\mathbf{v})=\frac{1}{2}\left(\frac{\partial v_{i}}{\partial x_{j}}+\frac{\partial v_{j}}{\partial x_{i}}\right) .
$$

Given a bounded domain $\Omega \subset \mathbb{R}^{n}, n \geq 2$, we say that the Korn inequality is valid in $\Omega$ for some $p, 1<p<\infty$, if for any $\mathbf{v} \in W^{1, p}(\Omega)^{n}$,

$$
\|\mathbf{v}\|_{W^{1, p}(\Omega)} \leq C\left\{\|\mathbf{v}\|_{L^{p}(\Omega)}+\|\varepsilon(\mathbf{v})\|_{L^{p}(\Omega)}\right\},
$$

where the constant $C$ depends only on $\Omega$ and $p$. Actually this is not the original statement of Korn (in particular, he considered only the case $p=2$ ), but his results can be derived in a simple way from this inequality. In what follows, if (1.1) is valid in $\Omega$ we will say shortly that $\Omega$ satisfies $\operatorname{Korn}_{p}$.

Received by the editors June 18, 2010.

2010 Mathematics Subject Classification. Primary 26D10; Secondary 76D07.

Key words and phrases. Korn inequality, divergence operator, bad domains.

This research was supported by ANPCyT under grants PICT 2006-01307 and 2007-910, by Universidad de Buenos Aires under grant X070, and by CONICET under grant PIP 11220090100625. The first and second authors are members of CONICET, Argentina. 
On the other hand, we will say that $\Omega$ satisfies $\operatorname{div}_{p}$ if, for any $f \in L_{0}^{p}(\Omega)$ (where the subscript 0 indicates that $\left.\int_{\Omega} f=0\right)$, there exists $\mathbf{u} \in W_{0}^{1, p}(\Omega)^{n}:=\overline{C_{0}^{\infty}(\Omega)^{n}}$ such that

$$
\operatorname{div} \mathbf{u}=f \quad \text { in } \Omega
$$

and

$$
\|\mathbf{u}\|_{W^{1, p}(\Omega)^{n}} \leq C\|f\|_{L^{p}(\Omega)}
$$

with $C$ depending only on $\Omega$ and $p$.

Since the pioneering works of Korn [K1, K2] and Friedrichs [F1, F2], there have been many papers analyzing the validity of $\operatorname{Korn}_{p}$ and $\operatorname{div}_{p}$ under different assumptions on the domains. Moreover, the relationship between both results has been widely studied (we refer to the review article $[\mathrm{H}$ and its references). In particular, it is well known that both results are valid for bounded Lipschitz domains and also for the broader class of John domains (see ADM, DRS). Concerning the relation between both results, it is known that, if $\Omega$ satisfies $\operatorname{div}_{p}$, then it also satisfies $\operatorname{Korn}_{p}$ (see for example ADM, BS, C1]).

Moreover, in the two-dimensional case, when $p=2$ and $\Omega$ is simply connected and with smooth boundary, a precise relation between the best constant in (1.1) and that in (1.2) was given in $\mathrm{HP}$. In that paper the authors considered also the following inequality for analytic functions due to Friedrichs [F1. Suppose that

$$
f(z)=\varphi(x, y)+i \psi(x, y)
$$

is an analytic function of the complex variable $z=x+i y$ in $\Omega \subset \mathbb{R}^{2}$, with $\varphi$ and $\psi$ real functions and $\int_{\Omega} \varphi=0$. Then, Friedrichs proved in [F1] that, under suitable assumptions on $\Omega$, there exists a constant $C$ depending only on $\Omega$, such that

$$
\|\varphi\|_{L^{2}(\Omega)} \leq C\|\psi\|_{L^{2}(\Omega)} .
$$

In $\mathrm{HP}$ the authors also obtained a relation between the best constant for this inequality and those in (1.1) and (1.2). It is not difficult to see that if $\Omega$ is simply connected, the Friedrichs inequality can be derived from both $\operatorname{div}_{2}$ or $\mathrm{Korn}_{2}$.

On the other hand, it is known that there are bounded domains which do not satisfy the inequalities introduced above. Indeed, a few counterexamples can be found in the literature. The oldest counterexample is due to Friedrichs [F1, who showed that the estimate (1.3) is not valid for some simply connected domains which have a quadratic external cusp (see [F1, page 343]). Another counterexample for the two-dimensional case was given in GG, again using domains with external cusps. For the three-dimensional case, a counterexample for the Korn inequality was given in $[\mathrm{W}]$.

In view of these counterexamples, a natural question is whether it is possible to obtain some weaker estimates similar to (1.2) and (1.1). Some results in this direction have been obtained in ADL, DL1, DL2 using weighted Sobolev norms. The weights used in those papers are powers of $d(x)$ (the distance of $x \in \Omega$ to $\partial \Omega$ ) for a general Hölder $\alpha$ domain, or powers of the distance to the set of singularities $M \subset \partial \Omega$, which we will denote with $d_{M}(x)$.

The goal of this paper is to present simple counterexamples for a class of domains with external cusps, and moreover to show that the results obtained for weighted norms in ADL, DL1, DL2 are optimal, in the sense that the powers of $d(x)$ or $d_{M}(x)$ involved in the estimates obtained in those papers cannot be improved. A particular case of our counterexamples was presented in [D]. 
Section 2 deals with the case of domains with a power type cusp. First, counterexamples for the Korn inequality (1.1) are given for planar domains. Then we recall some weighted Korn inequalities obtained in ADL, DL2 and use our examples to show that these results are optimal. Next we show that the techniques can be generalized to treat higher-dimensional domains with different kinds of power type singularities. We end this section showing that similar ideas can be applied to construct counterexamples for the related $\operatorname{div}_{p}$ problem and also to show that the weighted results given in [DL1, DL2] are optimal. Finally in Section 3, we extend the results for domains with more general cusps, i.e., cusps of nonpower type.

\section{Some Simple COUNTEREXAmples}

In this section we present very simple counterexamples for both problems $\operatorname{Korn}_{p}$ and $\operatorname{div}_{p}$ for cuspidal domains. As we will see, these counterexamples can also be used to prove optimality of the results in weighted norms obtained in ADL, DL1, DL2. For the sake of clarity we present first the results in two dimensions and explain afterwards how they can be extended for higher-dimensional domains.

For $\gamma>1$ consider the domain (see Figure 1)

$$
\Omega:=\left\{(x, y) \in \mathbb{R}^{2}: 0<x<1,|y|<x^{\gamma}\right\},
$$

and the vector field $\mathbf{w}=(u, v)=\left((s-1) y x^{-s}, x^{1-s}\right)$, with $s \in \mathbb{R}, s \neq 1$, to be chosen below.

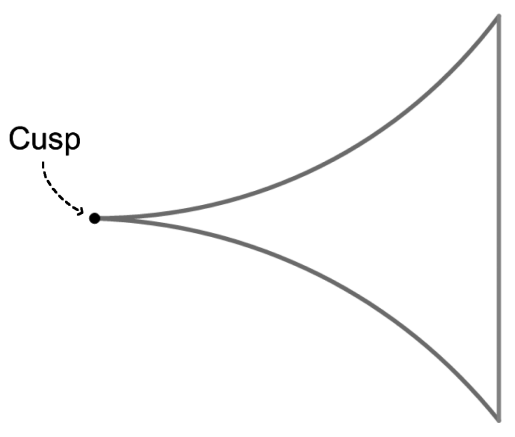

Figure 1. 2-Dimensional external cusp.

Let us notice that $\Omega$ is not a Lipschitz domain, but it is Hölder $\alpha$, with $\alpha=\frac{1}{\gamma}$. If $D \mathbf{w}$ is the differential matrix of $\mathbf{w}$ we have

$$
D \mathbf{w}=\left(\begin{array}{cc}
-s(s-1) y x^{-s-1} & (s-1) x^{-s} \\
(1-s) x^{-s} & 0
\end{array}\right)
$$

while, on the other hand,

$$
\varepsilon(\mathbf{w})=\left(\begin{array}{cc}
-s(s-1) y x^{-s-1} & 0 \\
0 & 0
\end{array}\right) .
$$


A straightforward computation shows that

$$
\begin{aligned}
\|\mathbf{w}\|_{L^{p}(\Omega)}^{p} & \leq C\left(\int_{0}^{1} \int_{0}^{x^{\gamma}}\left(y^{p} x^{-p s}+x^{p(1-s)}\right) d y d x\right) \\
& \leq C\left(\int_{0}^{1} \int_{0}^{x^{\gamma}} x^{p(1-s)} d y d x\right)=C \int_{0}^{1} x^{p-p s+\gamma} d x
\end{aligned}
$$

and

$$
\|\varepsilon(\mathbf{w})\|_{L^{p}(\Omega)}^{p} \leq C \int_{0}^{1} \int_{0}^{x^{\gamma}} y^{p} x^{-p(s+1)} d y d x \leq C \int_{0}^{1} x^{\gamma(p+1)-p(1+s)} d x ;
$$

hence

$$
s<\min \left\{\frac{(\gamma+1)}{p}+(\gamma-1), \frac{(\gamma+1)}{p}+1\right\} \Rightarrow\|\varepsilon(\mathbf{w})\|_{L^{p}(\Omega)^{p}},\|\mathbf{w}\|_{L^{p}(\Omega)^{p}}<\infty .
$$

However, we have

$$
\left\|\frac{\partial u}{\partial y}\right\|_{L^{p}(\Omega)}^{p}=C \int_{0}^{1} x^{-s p+\gamma} d x
$$

and so

$$
\left\|\frac{\partial u}{\partial y}\right\|_{L^{p}(\Omega)}<\infty \Rightarrow s<\frac{\gamma+1}{p} .
$$

But, since $\gamma>1$, it is possible to take $s$ such that

$$
\frac{\gamma+1}{p} \leq s<\min \left\{\frac{(\gamma+1)}{p}+(\gamma-1), \frac{(\gamma+1)}{p}+1\right\}
$$

and therefore, it follows from (2.5) and (2.6) that the classical Korn inequality (1.1) cannot be valid in $\Omega$.

Moreover, let us show that the same example can be used to show that the weighted results obtained for some nonsmooth domains in ADL, DL1, DL2] cannot be improved.

For example, for $\Omega \subset \mathbb{R}^{n}$, a Hölder $\alpha$ domain in any space dimension $n \geq 2$, the following weighted inequality holds for any $1<p<\infty$ [ADL, DL1,

$$
\left\|d^{1-\alpha} D \mathbf{w}\right\|_{L^{p}(\Omega)} \leq C\left\{\|\varepsilon(\mathbf{w})\|_{L^{p}(\Omega)}+\|\mathbf{w}\|_{L^{p}(B)}\right\},
$$

where $d$ stands for the distance to the boundary of $\Omega$ and $B$ is a fixed ball such that $\bar{B} \subset \Omega$. The same field $\mathbf{w}$ used in our counterexample for the classical inequality shows that (2.7) is sharp in the sense that the power of $d$ appearing on the left hand side cannot be reduced. Indeed, since the last term on the right hand side is the norm of $\mathbf{w}$ on the ball $B$ which satisfies $\bar{B} \subset \Omega$, the same computations given above now give

$$
s<\frac{(\gamma+1)}{p}+(\gamma-1) \Rightarrow\|\varepsilon(\mathbf{w})\|_{L^{p}(\Omega)},\|\mathbf{w}\|_{L^{p}(B)}<\infty
$$

instead of (2.5). Introducing the subdomain $\Omega_{\frac{1}{2}} \subset \Omega$,

$$
\Omega_{\frac{1}{2}}:=\left\{(x, y) \in \mathbb{R}^{2}: 0<x<1,0<|y|<\frac{1}{2} x^{\gamma}\right\},
$$


we have, for any $\beta$,

$$
\left\|d^{\beta} \frac{\partial u}{\partial y}\right\|_{L^{p}(\Omega)}^{p} \geq\left\|d^{\beta} \frac{\partial u}{\partial y}\right\|_{L^{p}\left(\Omega_{\frac{1}{2}}\right)}^{p} .
$$

Now, it can be easily checked that for our domain $\Omega, d(x, y) \simeq x^{\gamma}-|y|$ and therefore, for $(x, y) \in \Omega_{\frac{1}{2}}$, we obviously have $d(x, y) \simeq x^{\gamma}-|y| \geq \frac{1}{2} x^{\gamma}$. Hence

$$
\left\|d^{\beta} \frac{\partial u}{\partial y}\right\|_{L^{p}(\Omega)}^{p} \geq C \int_{0}^{1} \int_{0}^{x^{\gamma} / 2} x^{\beta \gamma p-s p} d y d x=C \int_{0}^{1} x^{\beta \gamma p-s p+\gamma} d x
$$

and so $\left\|d^{\beta} \frac{\partial u}{\partial y}\right\|_{L^{p}(\Omega)}$ is not finite if $\frac{\gamma+1}{p}+\beta \gamma \leq s$. On the other hand, for any $\beta<1-\alpha=1-\frac{1}{\gamma}$ it is possible to take $s$ such that

$$
\frac{\gamma+1}{p}+\beta \gamma \leq s<\frac{(\gamma+1)}{p}+(\gamma-1)
$$

which, in view of (2.8), shows the optimality of (2.7) in the sense that it is not possible to replace the power $1-\alpha$ on the left hand side by any power $\beta<1-\alpha$.

Powers of the distance to the boundary considered as weights seem a natural choice for general Hölder $\alpha$ domains since $\partial \Omega$ may have many singular points; in particular, the set of singular points can be a dense subset of $\partial \Omega$. However for cuspidal type domains, such as the one under consideration, powers of the distance to the cuspidal point might be used instead. In fact, the following result can be found in DL2]. For $\Omega$ as in (2.4), let $d_{M}$ be the distance to the cusp placed at $(0,0)$. Then

$$
\left\|d_{M}^{\gamma-1} D \mathbf{w}\right\|_{L^{p}(\Omega)} \leq C\left\{\|\varepsilon(\mathbf{w})\|_{L^{p}(\Omega)}+\|\mathbf{w}\|_{L^{p}(B)}\right\},
$$

where, as before, $B$ is a fixed ball satisfying $\bar{B} \subset \Omega$. Let us notice that for this particular domain, (2.10) is stronger than (2.7). Indeed, for $(x, y) \in \Omega$, we have $d_{M}(x, y)=\|(x, y)\| \simeq x$ and then $d^{1-\alpha}(x, y) \leq x^{\gamma(1-\alpha)} \simeq d_{M}^{\gamma-1}(x, y)$ while $d_{M}^{\gamma-1}(x, y)$ cannot be bounded by positive powers of $d(x, y)$. Moreover, it follows immediately that (2.10) cannot be improved by taking on the left hand side a smaller power of $d_{M}$. Indeed, since for any $\beta>0, d^{\alpha \beta} \leq d_{M}^{\beta}$ in $\Omega$, if we could replace $\gamma-1$ by some $\beta<\gamma-1$ in (2.10), we could also improve (2.7) by replacing $\alpha-1$ by $\alpha \beta<1-\alpha$.

Before proceeding, let us mention that both (2.7) and (2.10) can be generalized by taking some part of the weight to the right hand side. Indeed, in the first case it was proved in $\mathrm{ADL}$ that, for any $\beta$ such that $\alpha \leq \beta \leq 1$,

$$
\left\|d^{1-\beta} D \mathbf{w}\right\|_{L^{p}(\Omega)} \leq C\left\{\left\|d^{\alpha-\beta} \varepsilon(\mathbf{w})\right\|_{L^{p}(\Omega)}+\|\mathbf{w}\|_{L^{p}(B)}\right\}
$$

while in the second case (see [DL2]), for any $\beta \geq 0$,

$$
\left\|d_{M}^{\beta} D \mathbf{w}\right\|_{L^{p}(\Omega)} \leq C\left\{\left\|d_{M}^{\beta-\gamma+1} \varepsilon(\mathbf{w})\right\|_{L^{p}(\Omega)}+\|\mathbf{w}\|_{L^{p}(B)}\right\} .
$$

We have considered the particular cases (2.7) and (2.10) in order to simplify technical details. However, it can be seen that our counterexamples can be used to show that the above general estimates are also optimal.

On the other hand, a simple generalization of the vector field introduced for our counterexamples can be used to obtain similar results in arbitrary higher dimensions. Indeed, let us now define (see Figure 2),

$$
\Omega:=\left\{(x, \mathbf{y}) \in(0,1) \times \mathbb{R}^{n-1}:\|\mathbf{y}\|<x^{\gamma}\right\} .
$$




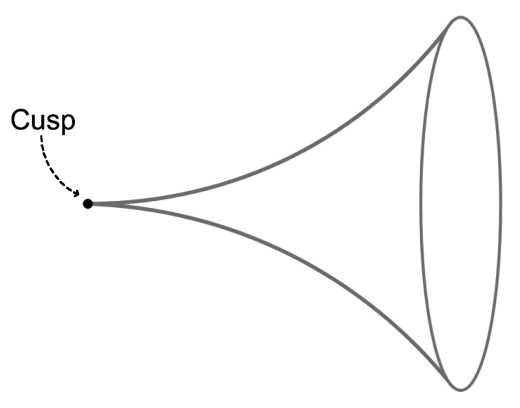

Figure 2. n-Dimensional external cusp.

Taking $\mathbf{w}=\left((s-1)\left(y_{1}+y_{2}+\cdots y_{n-1}\right) x^{-s}, x^{1-s}, \cdots, x^{1-s}\right)$ we have $D_{11} \mathbf{w}=-s(s-1)\left(y_{1}+\cdots+y_{n-1}\right) x^{-s-1}, \quad D_{1 j} \mathbf{w}=-D_{j 1} \mathbf{w}=(s-1) x^{-s}$ for $j>1$ and $D_{i j} \mathbf{w}=0$ otherwise. Therefore, the only nonzero component of $\varepsilon(\mathbf{w})$ is

$$
\varepsilon_{11}(\mathbf{w})=-s(s-1)\left(y_{1}+\cdots+y_{n-1}\right) x^{-s-1} .
$$

Then, a straightforward calculation yields

$$
\begin{aligned}
&\|\varepsilon(\mathbf{w})\|_{L^{p}(\Omega)}^{p}=C \int_{\Omega}\left|y_{1}+\cdots+y_{n-1}\right|^{p} x^{-p(s+1)} d \mathbf{y} d x \leq C \int_{\Omega}\|\mathbf{y}\|^{p} x^{-p(s+1)} d \mathbf{y} d x \\
& \leq C \int_{0}^{1} \int_{0}^{x^{\gamma}} \rho^{p+n-2} x^{-p(s+1)} d \rho d x \leq C \int_{0}^{1} x^{\gamma(p+n-1)-p(s+1)} d x \\
&\|\mathbf{w}\|_{L^{p}(\Omega)}^{p} \leq C \int_{\Omega} x^{(1-s) p} d \mathbf{y} d x \leq C \int_{0}^{1} x^{\gamma(n-1)+(1-s) p} d x
\end{aligned}
$$

and

$$
\|D \mathbf{w}\|_{L^{p}(\Omega)}^{p} \geq C \int_{\Omega} x^{-s p} d \mathbf{y} d x \geq C \int_{0}^{1} x^{\gamma(n-1)-s p} d x .
$$

Then, if

$$
\frac{\gamma(n-1)+1}{p} \leq s<\min \left\{\frac{\gamma(n-1)+1}{p}+\gamma-1, \frac{\gamma(n-1)+1}{p}+1\right\},
$$

$\|\varepsilon(\mathbf{w})\|_{L^{p}(\Omega)}$ and $\|\mathbf{w}\|_{L^{p}(\Omega)}$ are finite while $\|D \mathbf{w}\|_{L^{p}(\Omega)}$ is not. But, since $\gamma>1$, it is possible to choose $s$ satisfying (2.12), and therefore, (1.1) does not hold in $\Omega$.

Even more, similar arguments can be applied to show that the classic Korn inequality (1.1) is not valid in more general cusps. Indeed, for $k \geq 1$, define (see Figure 3)

$$
\Omega:=\left\{(x, \mathbf{y}, \mathbf{z}) \in(0,1) \times \mathbb{R}^{k} \times(0,1)^{n-k-1}:\|\mathbf{y}\|<x^{\gamma}\right\}
$$

We can generalize our counterexample by taking

$$
\mathbf{w}=((s-1)\left(y_{1}+y_{2}+\cdots y_{k}\right) x^{-s}, \overbrace{x^{1-s}, \cdots, x^{1-s}}^{k}, \overbrace{0, \cdots, 0}^{n-k-1}) .
$$




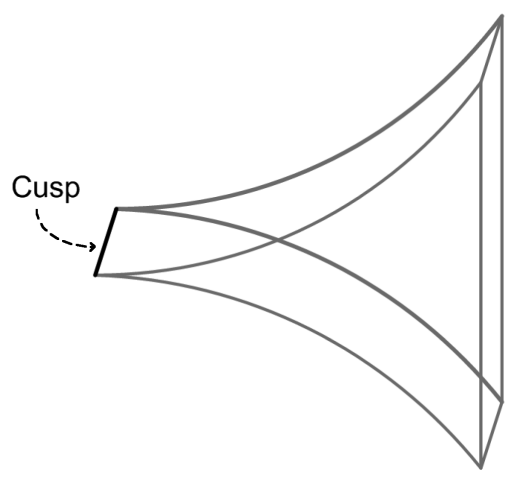

Figure 3. Flat cusp.

Now we have

$$
\begin{aligned}
\|\varepsilon(\mathbf{w})\|_{L^{p}(\Omega)}^{p} & =C \int_{\Omega}\left|y_{1}+\cdots+y_{k}\right|^{p} x^{-p(s+1)} d \mathbf{y} d x d \mathbf{z} \leq C \int_{\Omega}\|\mathbf{y}\|^{p} x^{-p(s+1)} d \mathbf{y} d x d \mathbf{z} \\
& \leq C \int_{0}^{1} \int_{0}^{x^{\gamma}} \rho^{p+k-1} x^{-p(s+1)} d \rho d x \leq C \int_{0}^{1} x^{\gamma(p+k)-p(s+1)} d x, \\
& \|\mathbf{w}\|_{L^{p}(\Omega)}^{p} \leq C \int_{\Omega} x^{(1-s) p} d \mathbf{y} d x \leq C \int_{0}^{1} x^{\gamma k+(1-s) p} d x,
\end{aligned}
$$

and

$$
\|D \mathbf{w}\|_{L^{p}(\Omega)}^{p} \geq \int_{\Omega} x^{-s p} d \mathbf{y} d x \geq C \int_{0}^{1} x^{\gamma k-s p} d x .
$$

Therefore, now taking $s$ such that

$$
\frac{\gamma k+1}{p} \leq s<\min \left\{\frac{\gamma k+1}{p}+\gamma-1, \frac{\gamma k+1}{p}+1\right\},
$$

which is possible for any $\gamma>1$, we conclude that (1.1) is not valid for the class of domains defined in (2.13).

Let us mention that the vector fields introduced for the counterexamples can be used to show the optimality of the powers in the weighted estimates (2.7) and (2.10) for the domains (2.11) and (2.13) (in this last case, and for (2.10), $d_{M}$ is defined as the distance to the singular set of the boundary placed at $(0, \mathbf{0}, \mathbf{z}), \mathbf{0}<\mathbf{z}<\mathbf{1})$.

As we have mentioned in the introduction, it is known that if a domain satisfies

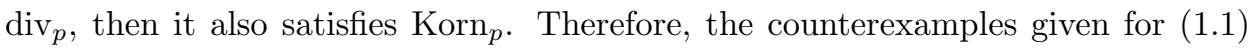
show immediately that the cuspidal domains defined above do not satisfy $\operatorname{div}_{p}$.

Although, in some particular cases, it was shown in DL2 that weighted Korn inequalities can be derived from weighted versions of $\operatorname{div}_{p}$, it is not clear whether this can be done in general.

However, it is not difficult to see by direct computations that the same vector fields introduced above can be used to show that cuspidal domains, such as those given in (2.11) and (2.13), do not satisfy $\operatorname{div}_{p}$. Moreover, as for the weighted Korn inequalities, it is possible to show the optimality of the weighted versions of $\operatorname{div}_{p}$ obtained in DL1, DL2.

Let us consider, for example, the weighted version of $\operatorname{div}_{p}$ proved in DL2]. To state a result obtained in that paper we first introduce some notation for weighted 
Sobolev spaces. Let $\omega, \omega_{1}$ and $\omega_{2}$ be nonnegative functions defined in a domain $\Omega$. For $1<p<\infty$, we denote with $L^{p}(\Omega, \omega)$ the Banach space with norm given by $\|f\|_{L^{p}(\Omega, \omega)}^{p}=\int_{\Omega}|f|^{p} \omega$ and define

$$
W^{1, p}\left(\Omega, \omega_{1}, \omega_{2}\right):=\left\{f \in L^{p}\left(\Omega, \omega_{1}\right): \quad \nabla f \in L^{p}\left(\Omega, \omega_{2}\right)^{n}\right\}
$$

and the corresponding norm,

$$
\|f\|_{W^{1, p}\left(\Omega, \omega_{1}, \omega_{2}\right)}^{p}:=\|f\|_{L^{p}\left(\Omega, \omega_{1}\right)}^{p}+\sum_{j=1}^{n}\left\|\frac{\partial f}{\partial x_{j}}\right\|_{L^{p}\left(\Omega, \omega_{2}\right)}^{p} .
$$

Also, we define $W_{0}^{1, p}\left(\Omega, \omega_{1}, \omega_{2}\right):=\overline{C_{0}^{\infty}(\Omega)}$, and if $\omega$ is such that $L^{p}(\Omega, \omega) \subset L^{1}(\Omega)$,

$$
L_{0}^{p}(\Omega, \omega):=\left\{f \in L^{p}(\Omega, \omega): \int_{\Omega} f=0\right\} .
$$

In what follows, $q$ will denote the dual exponent of $p$. For the family of domains defined in (2.13), and recalling that $d_{M}$ denotes the distance to the cusp, we have the following result proved in DL2, Theorem 4.1].

Theorem 2.1. Let $\Omega$ be as in (2.13) for a fixed $\gamma>1$, and $1<p<\infty$. If

$$
\beta \in\left(-\frac{\gamma(k+1)}{p}-\frac{\gamma-1}{q}, \frac{\gamma(k+1)}{q}-\frac{\gamma-1}{q}\right)
$$

and $\eta \geq \beta+\gamma-1$, then, for any $f \in L_{0}^{p}\left(\Omega, d_{M}^{p \beta}\right)$ there exists $\mathbf{w} \in W_{0}^{1, p}\left(\Omega, d_{M}^{p(\eta-1)}\right.$, $\left.d_{M}^{p \eta}\right)^{n}$ such that

$$
\operatorname{div} \mathbf{w}=f
$$

and

$$
\|\mathbf{w}\|_{W^{1, p}\left(\Omega, d_{M}^{p(\eta-1)}, d_{M}^{p \eta}\right)} \leq C\|f\|_{L^{p}\left(\Omega, d_{M}^{p \beta}\right)},
$$

where the constant $C$ depends only on $\beta, \eta, p$, and $\Omega$.

Remark 2.1. It is not difficult to check that the condition on $\beta$ in the statement of the theorem implies that $L^{p}\left(\Omega, d_{M}^{p \beta}\right) \subset L^{1}(\Omega)$, and therefore, $L_{0}^{p}\left(\Omega, d_{M}^{p \beta}\right)$ is well defined.

Let us show that the condition $\eta \geq \beta+\gamma-1$ in Theorem 2.1 is sharp. As in that theorem, we assume that $\beta$ satisfies (2.14). We will also assume that constant functions belong to $L^{p}\left(\Omega, d_{M}^{p \beta}\right)$, which leads to the condition

$$
0<\frac{\gamma k+1}{p}+\beta \text {. }
$$

For any $f \in L^{1}(\Omega)$ we denote with $f_{\Omega}$ its average over $\Omega$. Consider the function $f(x, \mathbf{y}, \mathbf{z})=x^{-s}$, where $0<s<\gamma k+1$ will be chosen below. Observe that, under this condition on $s$, we have $f \in L^{1}(\Omega)$ and so $f_{\Omega}$ is well defined.

Now, for $\epsilon>0$ small, we define

$$
f_{\epsilon}(x, \mathbf{y}, \mathbf{z})=\left\{\begin{array}{ccc}
f(x, \mathbf{y}, \mathbf{z}) & \text { if } \quad x>\epsilon \\
\epsilon^{-s} & \text { if } \quad x \leq \epsilon .
\end{array}\right.
$$

It follows from (2.15) that $f_{\epsilon} \in L^{p}\left(\Omega, d_{M}^{p \beta}\right)$.

Assume that the statement of Theorem 2.1 holds for some $\eta<\beta+\gamma-1$. Then, for each $\epsilon$, there exists $\mathbf{w}_{\epsilon}=\left(\mathrm{w}_{\epsilon, 1}, \mathbf{w}_{\epsilon, n-1}\right)$ such that $\operatorname{div} \mathbf{w}_{\epsilon}=f_{\epsilon}-f_{\epsilon, \Omega}$ and satisfying

$$
\left\|D \mathbf{w}_{\epsilon}\right\|_{L^{p}\left(\Omega, d_{M}^{p \eta}\right)} \leq C\left\|f_{\epsilon}-f_{\epsilon, \Omega}\right\|_{L^{p}\left(\Omega, d_{M}^{p \beta}\right)} .
$$


Then, recalling that $d_{M} \simeq x$, we have

$$
\begin{aligned}
\left\|f_{\epsilon}-f_{\epsilon, \Omega}\right\|_{L^{p}\left(\Omega, d_{M}^{p \beta}\right)}^{p} & \simeq \int_{\Omega}\left(f_{\epsilon}-f_{\epsilon, \Omega}\right)^{2}\left|f_{\epsilon}-f_{\epsilon, \Omega}\right|^{p-2} x^{p \beta} d x d \mathbf{y} d \mathbf{z} \\
& =\int_{\Omega} \operatorname{div} \mathbf{w}_{\epsilon}\left(f_{\epsilon}-f_{\epsilon, \Omega}\right)\left|f_{\epsilon}-f_{\epsilon, \Omega}\right|^{p-2} x^{p \beta} d x d \mathbf{y} d \mathbf{z},
\end{aligned}
$$

and therefore, integrating by parts we obtain

$$
\left\|f_{\epsilon}-f_{\epsilon, \Omega}\right\|_{L^{p}\left(\Omega, d_{M}^{p \beta}\right)}^{p} \simeq-\int_{\Omega} \mathrm{w}_{\epsilon, 1} \frac{\partial\left(\left(f_{\epsilon}-f_{\epsilon, \Omega}\right)\left|f_{\epsilon}-f_{\epsilon, \Omega}\right|^{p-2} x^{p \beta}\right)}{\partial x} d x d \mathbf{y} d \mathbf{z} .
$$

To simplify notation let us define

$$
h_{\epsilon}:=\frac{\partial\left(\left(f_{\epsilon}(x)-f_{\epsilon, \Omega}\right)\left|f_{\epsilon}(x)-f_{\epsilon, \Omega}\right|^{p-2} x^{p \beta}\right)}{\partial x} .
$$

Recalling that $(x, \mathbf{y}, \mathbf{z})=\left(x, y_{1}, \ldots, y_{k}, z_{1}, \ldots, z_{n-k-1}\right)$, we can write $h_{\epsilon}=\frac{\partial\left(y_{1} h_{\epsilon}\right)}{\partial y_{1}}$. Then, replacing in (2.17) and integrating by parts again, we obtain

$$
\left\|f_{\epsilon}-f_{\epsilon, \Omega}\right\|_{L^{p}\left(\Omega, d_{M}^{p \beta}\right)}^{p} \simeq \int_{\Omega} \frac{\partial \mathrm{w}_{\epsilon, 1}}{\partial y_{1}} y_{1} h_{\epsilon} d x d \mathbf{y} d \mathbf{z}
$$

But, it is not difficult to see that, for $x<f_{\Omega}^{-\frac{1}{s}} / 2$, we have

$$
\left|h_{\epsilon}\right| \leq C x^{-s(p-1)+p \beta-1} \text {. }
$$

Let us mention that we have considered $x$ small enough to be away from the point where $f_{\epsilon}(x)-f_{\epsilon, \Omega}=0$, because at that point $h_{\epsilon}$ blows up in the case $p<2$.

Then, applying the Hölder inequality in (2.18), we obtain

$$
\left\|f_{\epsilon}-f_{\epsilon, \Omega}\right\|_{L^{p}\left(\Omega, d_{M}^{p \beta}\right)}^{p} \leq C\left\|D \mathbf{w}_{\epsilon}\right\|_{L^{p}\left(\Omega, d_{M}^{p \eta}\right)}\left\|y_{1} x^{-s(p-1)+p \beta-1}\right\|_{L^{q}\left(\Omega, d_{M}^{-q \eta}\right)} .
$$

Therefore, using (2.16) and again $d_{M} \simeq x$, we conclude that

$$
\left\|f_{\epsilon}-f_{\epsilon, \Omega}\right\|_{L^{p}\left(\Omega, d_{M}^{p \beta}\right)}^{p} \leq C\left\|y_{1} x^{-s(p-1)+p \beta-1} x^{-\eta}\right\|_{L^{q}(\Omega)}^{q} .
$$

However, a straightforward computation shows that a choice of a positive $s$ in the range

$$
\frac{\gamma k+1}{p}+\beta \leq s<\frac{\gamma k+1}{p}+\beta+(\beta+\gamma-\eta-1) \frac{q}{p}
$$

leads to $\left\|f-f_{\Omega}\right\|_{L^{p}\left(\Omega, d_{M}^{p \beta}\right)}=+\infty$ and $\left\|y_{1} x^{-s(p-1)+p \beta-1} x^{-\eta}\right\|_{L^{q}(\Omega)}<\infty$. But, taking the limit $\epsilon \rightarrow 0$ in (2.19) we get a contradiction. Since $\eta<\beta+\gamma-1$ such an $s$ exists. Recall that we also need $s<\gamma k+1$, but, an $s$ satisfying this restriction and (2.20) exists because $\frac{\gamma k+1}{p}+\beta<\gamma k+1$. Indeed, this inequality follows from the assumption (2.14). Therefore, we conclude that a result such as that in Theorem 2.1 is not valid under this relation on the exponents.

To finish this section let us make some comments on solutions of the divergence for general Hölder- $\alpha$ domains. For this case, results analogous to Theorem 2.1, but with weights which are powers of the distance to the boundary, have been obtained in [DL1, DMRT]. Using arguments similar to those given above, one can show that the results obtained in those papers are optimal in the sense that the powers in the weighted estimates cannot be improved. We refer the reader to Theorem 4.2 in DL1, where a particular case was considered. 


\section{Domains with a general external Cusp}

In this section we consider the case of domains with more general external cusps. We have not been able to obtain a straightforward extension of the counterexamples given in the previous section, and therefore, we use here a different idea.

Let $\varphi:[0,1] \rightarrow \mathbb{R}$ be a $C^{1}$ function such that $\varphi(0)=0, \varphi_{x}(0)=0$ and $\varphi_{x}$ is strictly increasing. Two examples are

i) $\varphi(x)=x^{\gamma}$, with $\gamma>1$.

ii) $\varphi(x)=e^{-1 / x^{2}}$ in $(0,1]$ and $\varphi(0)=0$.

In particular, the arguments developed below give a different approach to construct counterexamples for the power type cusps considered in the previous section.

Associated with $\varphi$ we introduce the cuspidal domain $\Omega_{\varphi} \subset \mathbb{R}^{n}$ given by

$$
\Omega_{\varphi}=\left\{(x, \mathbf{y}, \mathbf{z}) \in(0,1) \times \mathbb{R}^{k} \times(0,1)^{n-k-1}:\|\mathbf{y}\|<\varphi(x)\right\},
$$

where $k \geq 1$.

Since $\varphi_{x}$ is a strictly increasing function, there is a monotone sequence $\left(x_{m}\right)_{m \geq m_{0}}$ $\subset(0,1]$ such that

$$
\varphi_{x}\left(x_{m}\right)=2^{-m} \text {. }
$$

We will also use the notation $r_{m}:=x_{m}-x_{m+1}$ and, without loss of generality, we assume that $m_{0}=1$ and $x_{1}=1$.

In the next lemma we prove two elementary properties of $\varphi(x)$.

Lemma 3.1. If $\left(x_{m}\right)_{m \geq 1}$ is the sequence defined in (3.2), then $\varphi$ satisfies:

$$
2^{-(m+2)} \leq \frac{\varphi(x)}{r_{m}} \quad \text { if } \quad \frac{x_{m+1}+x_{m}}{2} \leq x \leq x_{m}
$$

and

$$
\frac{\varphi(x)}{r_{m_{j}}} \leq 2^{-\left(m_{j}-1\right)} \quad \text { if } \quad x_{m_{j}+1} \leq x \leq x_{m_{j}},
$$

for an appropriate subsequence $\left(x_{m_{j}}\right)$ of $\left(x_{m}\right)$.

Proof. For $x \in\left(x_{m+1}, x_{m}\right]$ we have

$$
\begin{aligned}
\varphi(x) & =\varphi(x)-\varphi\left(x_{m+1}\right)+\varphi\left(x_{m+1}\right) \geq \varphi(x)-\varphi\left(x_{m+1}\right) \\
& =\varphi_{x}(\xi)\left(x-x_{m+1}\right) \geq 2^{-(m+1)}\left(x-x_{m+1}\right),
\end{aligned}
$$

where $\xi \in\left(x_{m+1}, x\right)$. So, if $x \in\left[\frac{x_{m+1}+x_{m}}{2}, x_{m}\right]$, it follows that $\left(x-x_{m+1}\right) \geq \frac{r_{m}}{2}$ and (3.3) is proved.

On the other hand, using that $\varphi(0)=0$ and an inductive argument, we have

$$
\begin{aligned}
\varphi\left(x_{m}\right) & \leq \varphi\left(x_{m+1}\right)+2^{-m}\left(x_{m}-x_{m+1}\right) \\
& \leq \varphi\left(x_{m+2}\right)+2^{-(m+1)}\left(x_{m+1}-x_{m+2}\right)+2^{-m}\left(x_{m}-x_{m+1}\right) \\
& \vdots \\
& \leq \sum_{i=m}^{\infty} 2^{-i}\left(x_{i}-x_{i+1}\right)=\sum_{i=m}^{\infty} 2^{-i} r_{i} .
\end{aligned}
$$


Now, we choose a subsequence $\left(r_{m_{j}}\right)$ of $\left(r_{m}\right)$ such that $\frac{r_{i}}{r_{m_{j}}} \leq 1$ for all $i \geq m_{j}$. For example, we can take $r_{m_{1}}$ as the maximum of $r_{i}$ over all $i$ and $r_{m_{j}}$ as the maximum of $r_{i}$ over all $i>m_{j-1}$. Then, it follows that

$$
\frac{\varphi\left(x_{m_{j}}\right)}{r_{m_{j}}} \leq \sum_{i=m_{j}}^{\infty} 2^{-i}=2^{-\left(m_{j}-1\right)}
$$

Therefore, using that $\varphi$ is increasing, we obtain (3.4).

In the next theorem we will prove some necessary conditions for weighted Korn type inequalities in $\Omega_{\varphi}$. Although generalizations for nonpower type $\varphi$ of the results given in ADL, DL1, DL2] have not been proved, we believe that powers of $\varphi_{x}$ are the natural weights to be considered. In the construction given in the following theorem we use some ideas from [Do].

Theorem 3.1. Let $\Omega_{\varphi} \subset \mathbb{R}^{n}$ be the domain defined in (3.1), $\beta_{1}, \beta_{2} \in \mathbb{R}, 1<p<\infty$ and $B$ a ball compactly contained in $\Omega_{\varphi}$. If there exists a positive constant $C$ such that

$$
\|D \mathbf{v}\|_{L^{p}\left(\Omega_{\varphi}, \varphi_{x}^{p \beta_{1}}\right)} \leq C\left\{\|\varepsilon(\mathbf{v})\|_{L^{p}\left(\Omega_{\varphi}, \varphi_{x}^{p \beta_{2}}\right)}+\|\mathbf{v}\|_{L^{p}(B)}\right\}
$$

for all $\mathbf{v} \in W^{1, p}\left(\Omega_{\varphi}, \varphi_{x}^{p \beta_{1}}\right)^{n}$, then $\beta_{1} \geq \beta_{2}+1$.

Proof. Let $\chi_{m}(x)$ be the characteristic function of the interval $\left[x_{m+1}, x_{m}\right]$. For each $m \geq 1$ we define $\mathbf{v}=(\mathrm{v}_{1}, \mathrm{v}_{2}, \ldots, \mathrm{v}_{k+1}, \overbrace{0, \cdots, 0}^{n-k-1}) \in W^{1, p}\left(\Omega_{\varphi}, \varphi_{x}^{p \beta_{1}}\right)^{n}$ (we omit the dependence on $m$ to simplify notation) by

$$
\mathrm{v}_{1}(x, \mathbf{y}, \mathbf{z})=\chi_{m}(x) \sin \left(\frac{2 \pi}{r_{m}}\left(x-x_{m+1}\right)\right) \frac{2 \pi}{r_{m}}\left(y_{1}+\cdots+y_{k}\right)
$$

and

$$
\mathrm{v}_{i}(x, \mathbf{y}, \mathbf{z})=\chi_{m}(x)\left(\cos \left(\frac{2 \pi}{r_{m}}\left(x-x_{m+1}\right)\right)-1\right)
$$

for $2 \leq i \leq k+1$.

It is easy to check that $\varepsilon_{i, j}(\mathbf{v})$ vanishes for $(i, j) \neq(1,1)$. So, since $B$ is compactly contained in $\Omega_{\varphi}$, it follows from (3.5) that

$$
\left\|\frac{\partial \mathrm{v}_{2}}{\partial x}\right\|_{L^{p}\left(\Omega_{\varphi}, \varphi_{x}^{p \beta_{1}}\right)} \leq C\left\|\varepsilon_{1,1}(\mathbf{v})\right\|_{L^{p}\left(\Omega_{\varphi}, \varphi_{x}^{p \beta_{2}}\right)}
$$

for $m$ sufficiently large. 
Now, using that the weight in the left hand side of (3.6) is equivalent to $2^{-m p \beta_{1}}$, if $x$ belongs to $\left[x_{m+1}, x_{m}\right]$, and property (3.3) we obtain

$$
\begin{aligned}
\left\|\frac{\partial \mathrm{v}_{2}}{\partial x}\right\|_{L^{p}\left(\Omega_{\varphi}, \varphi_{x}^{\left.p \beta_{1}\right)}\right.}^{p} & \simeq 2^{-m p \beta_{1}} \int_{\Omega_{\varphi}}\left|\sin \left(\frac{2 \pi}{r_{m}}\left(x-x_{m+1}\right)\right)\right|^{p}\left(\frac{2 \pi}{r_{m}}\right)^{p} \chi_{m}(x) d x d \mathbf{y} d \mathbf{z} \\
& \simeq \frac{2^{-m p \beta_{1}}}{r_{m}^{p-k-1}} \int_{x_{m+1}}^{x_{m}}\left|\sin \left(\frac{2 \pi}{r_{m}}\left(x-x_{m+1}\right)\right)\right|^{p}\left(\frac{\varphi(x)}{r_{m}}\right)^{k} \frac{2 \pi}{r_{m}} d x \\
& \geq \frac{2^{-m p \beta_{1}}}{r_{m}^{p-k-1}} \int_{\left(x_{m+1}+x_{m}\right) / 2}^{x_{m}}\left|\sin \left(\frac{2 \pi}{r_{m}}\left(x-x_{m+1}\right)\right)\right|^{p}\left(\frac{\varphi(x)}{r_{m}}\right)^{k} \frac{2 \pi}{r_{m}} d x \\
& \geq \frac{2^{-m\left(p \beta_{1}+k\right)}}{4^{k} r_{m}^{p-k-1}} \int_{\pi}^{2 \pi}|\sin t|^{p} d t \\
& \simeq \frac{2^{-m\left(p \beta_{1}+k\right)}}{r_{m}^{p-k-1}} .
\end{aligned}
$$

Analogously, if $m=m_{j}$ for some $j$, now using (3.4) we have

$$
\begin{aligned}
\left\|\varepsilon_{1,1}(\mathbf{v})\right\|_{L^{p}\left(\Omega_{\varphi}, \varphi_{x}^{p \beta_{2}}\right)}^{p} & \\
& \simeq 2^{-m p \beta_{2}} \int_{\Omega_{\varphi}}\left|\cos \left(\frac{2 \pi}{r_{m}}\left(x-x_{m+1}\right)\right)\left(\frac{2 \pi}{r_{m}}\right)^{2}\left(y_{1}+\cdots+y_{k}\right)\right|^{p} \chi(x) \\
& \simeq 2^{-m p \beta_{2}} \int_{x_{m+1}}^{x_{m}} \int_{0}^{\varphi(x)}\left|\cos \left(\frac{2 \pi}{r_{m}}\left(x-x_{m+1}\right)\right)\right|^{p}\left(\frac{2 \pi}{r_{m}}\right)^{2 p} \rho^{p+k-1} d \rho d x \\
& \simeq \frac{2^{-m p \beta_{2}}}{r_{m}^{p-k-1}} \int_{x_{m+1}}^{x_{m}}\left|\cos \left(\frac{2 \pi}{r_{m}}\left(x-x_{m+1}\right)\right)\right|^{p}\left(\frac{\varphi(x)}{r_{m}}\right)^{p+k} \frac{2 \pi}{r_{m}} d x \\
& \leq \frac{2^{-m\left(p \beta_{2}+p+k\right)}}{r_{m}^{p-k-1}} 2^{p+k} \int_{0}^{2 \pi}|\cos t|^{p} d t \\
& \simeq \frac{2^{-m\left(p \beta_{2}+p+k\right)}}{r_{m}^{p-k-1}} .
\end{aligned}
$$

Now, it follows from (3.6), (3.7) and (3.8) that there exists a positive constant $C$ which does not depend on $m$ such that

$$
\frac{2^{-m\left(p \beta_{1}+k\right)}}{r_{m}^{p-k-1}} \leq C \frac{2^{-m\left(p \beta_{2}+p+k\right)}}{r_{m}^{p-k-1}},
$$

for all $m=m_{j}$. Thus, we have $2^{-m_{j} p\left(\beta_{1}-\beta_{2}-1\right)} \leq C$ for all $j \geq 1$, and therefore, $\beta_{1} \geq \beta_{2}+1$ as we wanted to prove.

We end this section with an optimality result on solutions of the divergence in $\Omega_{\varphi}$. As in the previous theorem we will consider weights which are powers of $\varphi_{x}$.

Theorem 3.2. Let $\Omega_{\varphi} \subset \mathbb{R}^{n}$ be the domain defined in (3.1), $\beta_{1}, \beta_{2} \in \mathbb{R}$, and $1<p<\infty$. If for any $f \in L^{p}\left(\Omega_{\varphi}, \varphi_{x}^{p \beta_{2}}\right) \cap L_{0}^{1}\left(\Omega_{\varphi}\right)$ there exists $\mathbf{v} \in W_{0}^{1, p}\left(\Omega_{\varphi}, \varphi_{x}^{p \beta_{1}}\right)^{n}$ such that $\operatorname{div} \mathbf{v}=f$ and

$$
\|\mathbf{v}\|_{W_{0}^{1, p}\left(\Omega_{\varphi}, \varphi_{x}^{p \beta_{1}}\right)} \leq C\|f\|_{L^{p}\left(\Omega_{\varphi}, \varphi_{x}^{p \beta_{2}}\right)},
$$

where $C$ depends only on $\Omega_{\varphi}, \beta_{1}, \beta_{2}$ and $p$, then $\beta_{1} \geq \beta_{2}+1$. 
Proof. We will use again the sequence $x_{m}$ introduced in (3.2). Defining $a_{m}=$ $\left(x_{m+1}+x_{m}\right) / 2$, we introduce for each $m \in \mathbb{N}$ the domain

$$
\Omega_{m}=\left\{\left(2 a_{m}-x, \mathbf{y}, \mathbf{z}\right):(x, \mathbf{y}, \mathbf{z}) \in \Omega_{\varphi}\right\} .
$$

Observe that $\Omega_{m}$ is obtained from $\Omega_{\varphi}$ by symmetry and translation. Therefore, under the hypothesis of the theorem we know that, for any $g \in L^{p}\left(\Omega_{m}, \omega_{2}\right) \cap L_{0}^{1}\left(\Omega_{\varphi}\right)$, there exists $\mathbf{v} \in W_{0}^{1, p}\left(\Omega_{m}, \omega_{1}\right)^{n}$ such that $\operatorname{div} \mathbf{v}=g$ and

$$
\|\mathbf{v}\|_{W_{0}^{1, p}\left(\Omega_{m}, \omega_{1}\right)} \leq C\|g\|_{L^{p}\left(\Omega_{m}, \omega_{2}\right)}
$$

where $\omega_{1}(x)=\varphi_{x}^{p \beta_{1}}\left(2 a_{m}-x\right)$ and $\omega_{2}(x)=\varphi_{x}^{p \beta_{2}}\left(2 a_{m}-x\right)$. It is important to remark that the constant in (3.10) is equal to the one in (3.9), and therefore independent of $m$.

Now, let us define $U_{m} \subset \Omega_{\varphi}$ and $U_{m}^{\prime} \subset \Omega_{m}$ as

$$
\begin{aligned}
& U_{m}=\left\{(x, \mathbf{y}, \mathbf{z}) \in \Omega_{\varphi}: x_{m+1}<x<x_{m}\right\}, \\
& U_{m}^{\prime}=\left\{(x, \mathbf{y}, \mathbf{z}) \in \Omega_{m}: x_{m+1}<x<x_{m}\right\},
\end{aligned}
$$

and

$$
D_{m}=U_{m} \cup U_{m}^{\prime}
$$

Observe that $D_{m}$ is a Lipschitz domain. Recalling the definition of $x_{m}$ and that $\varphi_{x}$ is an increasing function, we have that, in $D_{m}, \omega_{1} \simeq 2^{-m p \beta_{1}}$ and $\omega_{2} \simeq 2^{-m p \beta_{2}}$. Moreover, $D_{m}$ is symmetric with respect to $x=a_{m}$.

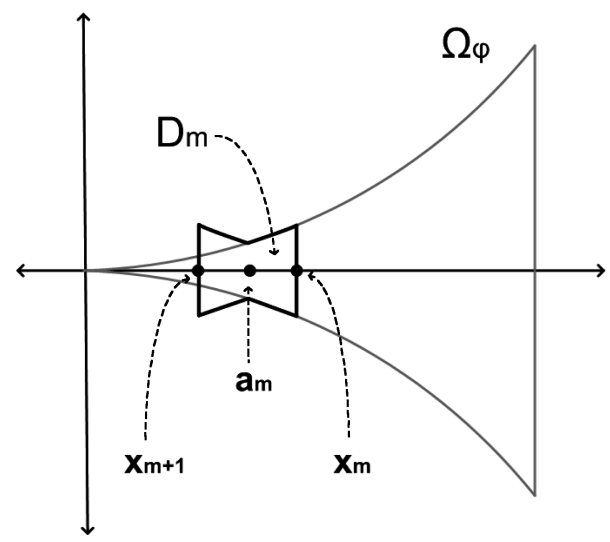

Figure 4. The Lipschitz domains $\mathbf{D}_{m}$

Using an argument introduced in $\left[\mathrm{B}\right.$, we can decompose any $g \in L_{0}^{p}\left(D_{m}\right)$ as a sum of functions $g_{1}, g_{2} \in L_{0}^{p}\left(D_{m}\right)$, where $g_{1}$ and $g_{2}$ are supported in $U_{m}$ and $U_{m}^{\prime}$ respectively. In fact, we define

$$
g_{1}(x, \mathbf{y}, \mathbf{z})=\left\{\begin{array}{cl}
g(x, \mathbf{y}, \mathbf{z})-\frac{\chi(x, \mathbf{y}, \mathbf{z})}{\left|U_{m} \cap U_{m}^{\prime}\right|} \int_{U_{m}} g & \text { in } U_{m}, \\
0 & \text { in } D_{m} \backslash U_{m}
\end{array}\right.
$$

where $\chi$ is the characteristic function of $U_{m} \cap U_{m}^{\prime}$, and $g_{2}:=g-g_{1}$. Moreover, we extend $g_{1}$ and $g_{2}$ by zero outside $D_{m}$. 
Then, using the Hölder inequality, we have

$$
\begin{aligned}
\left\|g_{1}\right\|_{L^{p}\left(\Omega_{\varphi}, \varphi_{x}^{\left.p \beta_{2}\right)}\right.} & \simeq 2^{-m \beta_{2}}\left\|g_{1}\right\|_{L^{p}\left(D_{m}\right)} \leq 2^{-m \beta_{2}}\left(\|g\|_{L^{p}\left(D_{m}\right)}+\frac{1}{\left|U_{m} \cap U_{m}^{\prime}\right|^{1 / q}} \int_{U_{m}}|g|\right) \\
& \leq 2^{-m \beta_{2}}\|g\|_{L^{p}\left(D_{m}\right)}\left(1+\frac{\left|U_{m}\right|^{1 / q}}{\left|U_{m} \cap U_{m}^{\prime}\right|^{1 / q}}\right) .
\end{aligned}
$$

If $m=m_{j}$ for some $j$, it is easy to check, using Lemma 3.1 that

$$
1 \leq \frac{\left|U_{m}\right|}{\left|U_{m} \cap U_{m}^{\prime}\right|} \leq C
$$

where the constant $C$ does not depend on $m$. Thus, we have

$$
\left\|g_{1}\right\|_{L^{p}\left(\Omega_{\varphi}, \varphi_{x}^{p \beta_{2}}\right)} \leq C 2^{-m \beta_{2}}\|g\|_{L^{p}\left(D_{m}\right)},
$$

and consequently,

$$
\left\|g_{2}\right\|_{L^{p}\left(\Omega_{m}, \omega_{2}\right)} \simeq 2^{-m \beta_{2}}\left\|g_{2}\right\|_{L^{p}\left(D_{m}\right)} \leq C 2^{-m \beta_{2}}\|g\|_{L^{p}\left(D_{m}\right)} .
$$

Now, from (3.9), (3.10), (3.11), and (3.12) it follows that, for any $g \in L_{0}^{p}\left(D_{m}\right)$, there exists $\mathbf{u} \in W^{1, p}\left(D_{m}\right)^{n}$ such that $\operatorname{div} \mathbf{u}=g, \mathbf{u}=0$ in $\partial D_{m} \cap\left\{\left|x-a_{m}\right| \neq r_{m} / 2\right\}$ and

$$
\|\mathbf{u}\|_{W^{1, p}\left(D_{m}\right)} \leq C 2^{-m\left(\beta_{2}-\beta_{1}\right)}\|g\|_{L^{p}\left(D_{m}\right)} .
$$

Now, take $g \in L_{0}^{p}\left(D_{m}\right)$ and $\mathbf{u}$ as above and let $f \in L^{q}\left(D_{m}\right)$ be defined by $f(x, \mathbf{y}, \mathbf{z})=\sin \left(\frac{2 \pi}{r_{m}}\left(x-a_{m}\right)\right)$. Then, integrating by parts and using (3.13), we have

$$
\begin{aligned}
\int_{D_{m}} f g & =\int_{D_{m}} f \operatorname{div} \mathbf{u} \\
& =-\int_{D_{m}} \frac{2 \pi}{r_{m}} \cos \left(\frac{2 \pi}{r_{m}}\left(x-a_{m}\right)\right) \mathrm{u}_{1}+\int_{\partial D_{m}} \overbrace{\sin \left(\frac{2 \pi}{r_{m}}\left(x-a_{m}\right)\right)}^{=0} \mathbf{u} \cdot \nu \\
& =-\frac{2 \pi}{r_{m}} \int_{D_{m}} \frac{\partial y_{1}}{\partial y_{1}} \cos \left(\frac{2 \pi}{r_{m}}\left(x-a_{m}\right)\right) \mathrm{u}_{1} \\
& =\frac{2 \pi}{r_{m}} \int_{D_{m}} y_{1} \cos \left(\frac{2 \pi}{r_{m}}\left(x-a_{m}\right)\right) \frac{\partial \mathrm{u}_{1}}{\partial y_{1}}-\frac{2 \pi}{r_{m}} \int_{\partial D_{m}} y_{1} \cos \left(\frac{2 \pi}{r_{m}}\left(x-a_{m}\right)\right) \overbrace{\mathrm{u}_{1} \nu_{2}} \\
& \leq \frac{2 \pi}{r_{m}}\left\|y_{y_{1} \cos }\left(\frac{2 \pi}{r_{m}}\left(x-a_{m}\right)\right)\right\|_{L^{q}\left(D_{m}\right)} \| \mathbf{u}_{L^{p}\left(D_{m}\right)} \\
& \leq C \frac{2^{-m\left(\beta_{2}-\beta_{1}\right)}}{r_{m}}\left\|y_{1} \cos \left(\frac{2 \pi}{r_{m}}\left(x-a_{m}\right)\right)\right\|_{L^{q}\left(D_{m}\right)}\|g\|_{L^{p}\left(D_{m}\right)} .
\end{aligned}
$$

But, since $f$ and $D_{m}$ are symmetric with respect to $x=a_{m}$, we have $\int_{D_{m}} f=0$, and so

$$
\|f\|_{L^{q}\left(D_{m}\right)}=\sup _{0 \neq g \in L_{0}^{p}\left(D_{m}\right)} \frac{\int_{D_{m}} f g}{\|g\|_{L^{p}\left(D_{m}\right)}} \leq C \frac{2^{-m\left(\beta_{2}-\beta_{1}\right)}}{r_{m}}\left\|y_{1} \cos \left(\frac{2 \pi}{r_{m}}\left(x-a_{m}\right)\right)\right\|_{L^{q}\left(D_{m}\right)} .
$$


Finally, computing the norms we obtain that, for all $m=m_{j}$,

$$
r_{m}^{(k+1) / q} 2^{-m k / q} \leq C \frac{2^{-m\left(\beta_{2}-\beta_{1}\right)}}{r_{m}} r_{m}^{(q+k+1) / q} 2^{-m(k+q) / q},
$$

and therefore,

$$
2^{-m\left(\beta_{1}-\beta_{2}-1\right)} \leq C
$$

Thus $\beta_{1} \geq \beta_{2}+1$.

Remark 3.1. In the particular case $\beta_{2}=0$, the result in Theorem 3.2 can be deduced from Theorem 3.1 with $\beta_{1}=0$. Indeed, this follows from [DL2, Theorem 6.1].

\section{REFERENCES}

[ADL] G. Acosta, R. Durán and A. Lombardi, Weighted Poincaré and Korn inequalities for Hölder $\alpha$ domains, Math. Meth. Appl. Sci. (MMAS) 29 (4), pp. 387-400, 2006. MR.2198138 (2006i:26020)

[ADM] G. Acosta, R. G. Durán and M. A. Muschietti, Solutions of the divergence operator on John domains, Advances in Mathematics 206(2), pp. 373-401, 2006. MR2263708 (2007k:35035)

[B] M. E. Bogovskii, Solution of the first boundary value problem for the equation of continuity of an incompressible medium, Soviet Math. Dokl. 20, pp. 1094-1098, 1979. MR.553920 (82b:35135)

[BS] S. C. Brenner and L. R. Scott, The Mathematical Theory of Finite Element Methods, Springer-Verlag, Berlin, 1994. MR,1278258 (95f:65001)

[C1] P. G. Ciarlet, Introduction to linear shell theory, Series in Applied Mathematics, P. G. Ciarlet and P. L. Lions, eds., Gauthier-Villars, 1998. MR.1648549 (99j:73071)

[DRS] L. Diening, M. Ružička and K. Schumacher, A decomposition technique for John domains, Annales Academiae Scientiarum Fennicae Ser. A I Math. 35, pp. 87-114, 2010. MR.2643399(2011i:26020)

[Do] M. Dobrowolski, On the LBB condition in the numerical analysis of the Stokes equations, Appl. Numer. Math. 54, pp. 314-323, 2005. MR2149355(2006d:65132)

[D] R. G. Durán, The inf-sup condition for the Stokes equations: A constructive approach in general domains, Mathematisches Forschungsinstitut Oberwolfach, Workshop on Gemischte und nicht-standard Finite-Elemente-Methoden mit Anwendungen, Extended abstract 5, pp. 270-272, 2005.

[DMRT] R. G. Durán, M.-A. Muschietti, E. Russ, and P. Tchamitchian, Divergence operator and Poincaré inequalities on arbitrary bounded domains, Complex Variables and Elliptic Equations 55, pp. 795-816, 2010. MR2674865 (2011e:35056)

[DL1] R. Durán and F. Lopez García, Solutions of the divergence and analysis of the Stokes equations in planar Hölder- $\alpha$ domains, Math. Mod. Meth. Appl. Sci. 20(1), pp. 95-120, 2010. MR.2606245 (2011h:35219)

[DL2] R. Durán and F. Lopez García, Solutions of the divergence and Korn inequalities on domains with an external cusp, Annales Academiae Scientiarum Fennicae Ser. A I Math. 35, pp. 421-438, 2010. MR2731700 (2011k:35180)

[F1] K. O. Friedrichs, On certain inequalities and characteristic value problems for analytic functions and for functions of two variables, Trans. Amer. Math. Soc., 41, pp. 321-364, 1937. MR 1501907

[F2] K. O. Friedrichs, On the boundary-value problems of the theory of elasticity and Korn's inequality, Ann. of Math. (2), 48, pp. 441-471, 1947. MR0022750 (9:255b)

[GG] G. Geymonat and G. Gilardi, Contre-exemples à l'inégalité de Korn et au Lemme de Lions dans des domaines irréguliers, Equations aux Dérivées Partielles et Applications, Gauthiers-Villars, pp. 541-548, 1998. MR.1648239 (99i:73017)

[H] C. O. Horgan, Korn's inequalities and their applications in continuum mechanics, SIAM Review, 37, pp. 491-511, 1995. MR1368384 (96h:73014)

[HP] C. O. Horgan and L. E. Payne, On inequalities of Korn, Friedrichs and Babuska-Aziz, Arch. Rational Mech. Anal. 82, pp. 165-179, 1983. MR687553 (84d:73014) 
[K1] A. Korn, Die Eigenschwingungen eines elastischen Korpers mit ruhender Oberflache, Akad. der Wissensch Munich, Math-phys. Kl, Beritche, 36, pp. 351-401, 1906.

[K2] A. Korn, Ubereinige Ungleichungen, welche in der Theorie der elastischen und elektrischen Schwingungen eine Rolle spielen, Bulletin Internationale, Cracovie Akademie Umiejet, Classe de sciences mathématiques et naturelles, pp. 705-724, 1909.

[W] N. Weck, Local compactness for elasticity in irregular domains, Math. Meth. in Appl. Sci. 17, pp. 107-113, 1994. MR1258259 (95a:46056)

Departamento de Matemática and imas, COniCET, Facultad de Ciencias Exactas y Naturales, Universidad de Buenos Aires, 1428 Buenos Aires, Argentina

E-mail address: gacosta@dm.uba.ar

Departamento de Matemática and imas, COniCET, Facultad de Ciencias Exactas y Naturales, Universidad de Buenos Aires, 1428 Buenos Aires, Argentina

E-mail address: rduran@dm.uba.ar

Departamento de Matemática, Facultad de Ciencias Exactas y Naturales, UniversiDad de Buenos Aires, 1428 Buenos Aires, Argentina

E-mail address: flopezg@dm.uba.ar

Current address: Worcester Polytechnic Institute, 100 Institute Road, Worcester, Massachusetts 01609-2280

E-mail address: flopezgarcia@wpi.edu 